\title{
Dynamic characteristics of ultra-precision aerostatic bearings
}

\author{
Xue-Dong Chen $\cdot$ Jin-Cheng Zhu $\cdot$ Han Chen
}

Received: 3 October 2012/ Accepted: 12 November 2012/Published online: 13 March 2013

(C) Shanghai University and Springer-Verlag Berlin Heidelberg 2013

\begin{abstract}
With high acceleration and ultra-precision requirements, the design of aerostatic bearings has been gradually focused on their dynamic performances. In this paper, the dynamic stiffness and damping coefficients of aerostatic bearings are investigated. Due to compressibility of the gas, the dynamic characteristics of aerostatic bearings show nonlinear frequency dependence. Particularly, their nonlinear dynamic behaviors are quite remarkable for ultra-precision aerostatic bearings with small air gap heights and high supply pressure.
\end{abstract}

Keywords Aerostatic bearing - Dynamic characteristics . Dynamic mesh

\section{Introduction}

Recently, aerostatic bearings are increasingly utilized in ultra-precision machine tools and semiconductor manufacturing equipments. With increasing requirements such as higher acceleration and smaller vibration, traditional static characteristics, such as load carrying capacity and static stiffness, are no longer sufficient in the design of ultra-precision aerostatic bearings. Instead, dynamic performances become increasingly critical and should be considered in the design of ultra-precision aerostatic bearings.

Due to squeeze film effects, aerostatic bearings show complex dynamic behaviors when they are subjected to

X.-D. Chen $(\bowtie) \cdot$ J.-C. Zhu $\cdot$ H. Chen

State Key Laboratory of Digital Manufacturing Equipment and

Technology, Huazhong University of Science and Technology,

Wuhan 430074, People's Republic of China

e-mail: chenxd@mail.hust.edu.cn dynamic impact loads. The dynamic instability of the air bearing, e.g., self-excited vibration ("pneumatic hammer"), was considered in early studies [1]. For high speed moving applications, some new configuration designs were proposed to improve dynamic stiffness and damping coefficients of aerostatic bearings [2-4]. There were also qualitative analyses of the compressible thin air films in gas lubricated components, which indicated the dependence of their dynamic characteristics on the excitation frequency [5].

Generally, analytical solutions of the dynamic pressure distribution in aerostatic bearings, especially those with complex geometrical configurations, are impossible to obtain. Numerical techniques, such as finite difference method (FDM) [4] and finite element method (FEM) [6], have been adopted in the study of dynamic characteristics of aerostatic bearings. In this paper, a dynamic mesh modeling technique using commercial CFD software is proposed to investigate dynamic characteristics of multirestrictor aerostatic bearings with shallow recesses. The focus of this work is on the influences of excitation magnitude and frequency on the dynamic stiffness and damping coefficients of aerostatic bearings with different air gap heights and supply pressure values.

\section{Aerostatic bearing and theoretical model}

A circular pad aerostatic thrust bearing is studied, as shown in Fig. 1. The bearing pad has an outer diameter $d_{2}=77 \mathrm{~mm}$ and four supply orifice restrictors equally spaced along a circumference of $50 \mathrm{~mm}$ diameter. All restrictors are identical, and each of them has a shallow recess used to improve static performances of the bearing. The orifice diameter is $d_{0}=0.3 \mathrm{~mm}$. The diameter and the 


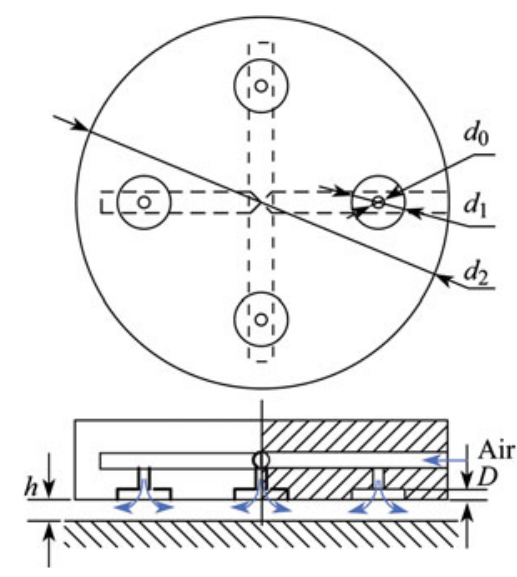

Fig. 1 Sketch of the aerostatic bearing

depth of each cylindrical recess are $d_{1}=5 \mathrm{~mm}$ and $D=0.15 \mathrm{~mm}$, respectively. The small air gap height on the order of $10 \mu \mathrm{m}$ is considered, so that high enough stiffness of the bearing can be achieved in ultra precision applications.

Pressure distribution of the air flowing in the bearing gap can be determined from the Reynolds equation as follows

$\frac{\partial}{\partial x}\left(\rho h^{3} \frac{\partial p}{\partial x}\right)+\frac{\partial}{\partial y}\left(\rho h^{3} \frac{\partial p}{\partial y}\right)+12 \mu q \delta=12 \mu \frac{\partial}{\partial t}(\rho h)$,

where $p$ is the air pressure in the gap, $\rho$ the air density, $\mu$ dynamic viscosity of the air, $q$ the mass flow rate, and $\delta$ Kronecker delta (at orifice inlet, $\delta=1$; otherwise, $\delta=0$ ).

When the air gap height is excited with a small disturbance as $h=h_{0}+\Delta h=h_{0}+h_{1} e^{j \omega t}\left(j=\sqrt{-1}, h_{1} \ll h_{0}\right)$, the resulting pressure in the air film is also disturbed as $p=p_{0}+\Delta p=p_{0}+p_{1} e^{j \omega t}$ due to the well-known "squeeze film effects". Based on the perturbation theory, the perturbed form of (1) can be written as

$$
\begin{aligned}
& 2\left(\frac{\partial}{\partial x}\left(h_{0}^{3} \frac{\partial}{\partial x}\left(p_{0} \frac{\Delta p}{\Delta h}\right)\right)+\frac{\partial}{\partial y}\left(h_{0}^{3} \frac{\partial}{\partial y}\left(p_{0} \frac{\Delta p}{\Delta h}\right)\right)\right) \\
& \quad+24 \mu R T \delta \frac{\partial q}{\partial p} \frac{\Delta p}{\Delta h}+3\left(\frac{\partial}{\partial x}\left(h_{0}^{2} \frac{\partial p_{0}^{2}}{\partial x}\right)+\frac{\partial}{\partial y}\left(h_{0}^{2} \frac{\partial p_{0}^{2}}{\partial y}\right)\right) \\
& \quad=\mathrm{j} 24 \mu\left(h_{0} \frac{\Delta p}{\Delta h} \omega+p_{0} \omega\right)
\end{aligned}
$$

Comparing (2) with its incompressible counterpart (3), it is apparent that the pressure and gap height variation is nonlinear due to the additional nonlinear term on the right hand side of (2).

$$
\begin{gathered}
\left(\frac{\partial}{\partial x}\left(h_{0}^{3} \frac{\partial}{\partial x}\left(\frac{\Delta p}{\Delta h}\right)\right)+\frac{\partial}{\partial y}\left(h_{0}^{3} \frac{\partial}{\partial y}\left(\frac{\Delta p}{\Delta h}\right)\right)\right)+\delta \frac{12 \mu}{\rho} \frac{\partial q}{\partial p} \frac{\Delta p}{\Delta h} \\
+3\left(\frac{\partial}{\partial x}\left(h_{0}^{2} \frac{\partial p_{0}}{\partial x}\right)+\frac{\partial}{\partial y}\left(h_{0}^{2} \frac{\partial p_{0}}{\partial y}\right)\right)=\mathrm{j} 12 \mu \omega .
\end{gathered}
$$

Furthermore, the solution of (2) can be represented as

$$
\frac{\Delta p}{\Delta h}=\operatorname{Re}\left(\omega, p_{0}, h_{0}\right)+\mathrm{j} \operatorname{Im}\left(\omega, p_{0}, h_{0}\right),
$$

where Re denotes the real part, and Im denotes the imaginary part. Apparently, they are nonlinearly frequency-dependent and can be influenced by the initial conditions, $p_{0}$ and $h_{0}$.

From (4), the complex stiffness of the aerostatic bearing can be calculated as

$K^{*}=\int \operatorname{Re}\left(\omega, p_{0}, h_{0}\right) \mathrm{d} A+\mathrm{j} \int \operatorname{Im}\left(\omega, p_{0}, h_{0}\right) \mathrm{d} A$,

where the real part denotes the stiffness coefficient $K$, while the imaginary part is the product of the damping coefficient $C$ and excitation frequency $\omega$, i.e.

$K=\int \operatorname{Re}\left(\omega, p_{0}, h_{0}\right) \mathrm{d} A, \quad C=\int \operatorname{Im}\left(\omega, p_{0}, h_{0}\right) / \omega \mathrm{d} A$.

Accordingly, with the effects of air compressibility, the dynamic coefficients $K$ and $C$ of aerostatic bearings are also nonlinearly frequency-dependent. Moreover, they will vary with different air gap heights and supply pressures.

\section{Numerical simulation and validation}

The commercial CFD software ANSYS-Fluent is adopted to numerically study the dynamic characteristics of the aerostatic bearing. According to symmetry of the geometry and boundary conditions, only $1 / 8$ of the 3D air flow field in the aerostatic bearing is considered, and the computational meshes are shown in Fig. 2. There are totally 135,510 hexahedral control volumes in the computational domain. Pressure inlet and pressure outlet boundary conditions are specified at the orifice inlet and the bearing periphery, respectively. The two radial boundaries are symmetric, and adiabatic boundary conditions are used on the solid walls. Since the Knudsen number based on the air film height is no more than 0.01, slip effects can be ignored [7] and no-slip boundary conditions are used on the solid walls. The dynamic mesh modeling approach is employed to solve for the time-dependent pressure field in the aerostatic bearing. In this study, the bottom wall of the air gap is specified as a sinusoidally moving boundary (displacement excitation), and the adjacent control volumes can deform accordingly.

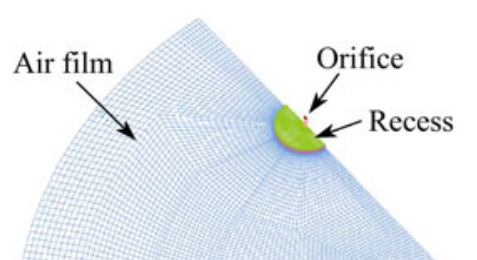

Fig. 2 Computational model of the aerostatic bearing 




Fig. 3 Test setup



Fig. 4 Static load varies with air gap height

In order to validate the CFD model of the aerostatic bearing, a static load experiment was performed, and the test setup is displayed in Fig. 3. Figure 4 shows the variation of the static load with the air gap height, in which it can be seen that the simulation results agree with the experimental data very well.

\section{Results and discussion}

From the theoretical analysis, the pressure variation in the aerostatic bearing is related to the excitation frequency of the air gap height, and so is the dynamic load. Figure 5 plots the numerically computed variations of the bearing load in ten periods under three different excitations, whose amplitudes are identical while frequencies are 10, 100 and 1,000 Hz. From Fig. 5, it can be seen that the amplitude of the dynamic load increases significantly with increasing excitation frequency, and the phase of the response curve also shifts with the excitation frequency. This conclusion is also consistent with that in the squeeze film damping analysis.

Dynamic stiffness and damping coefficients of the aerostatic bearing are plotted in Fig. 6 as functions of the excitation frequency, where the air gap height is $h$ equal to $10 \mu \mathrm{m}$ and the air supply pressure is $P_{\mathrm{s}}=0.4 \mathrm{MPa}$.

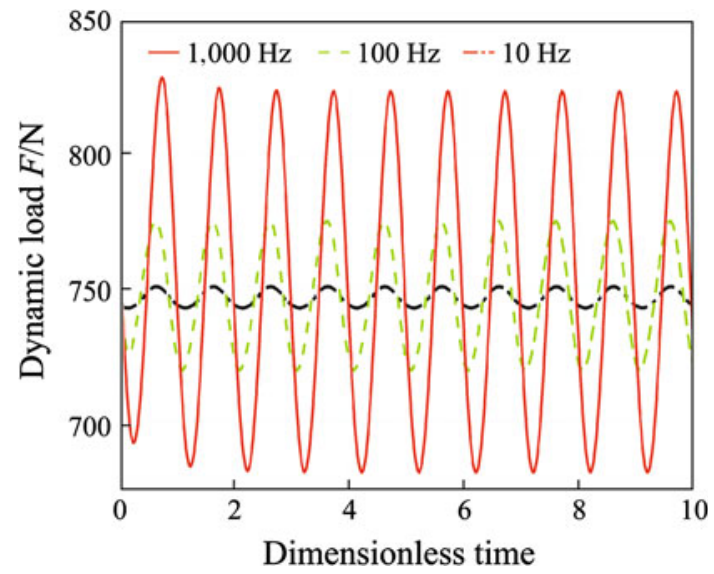

Fig. 5 Variations of dynamic load with different frequencies

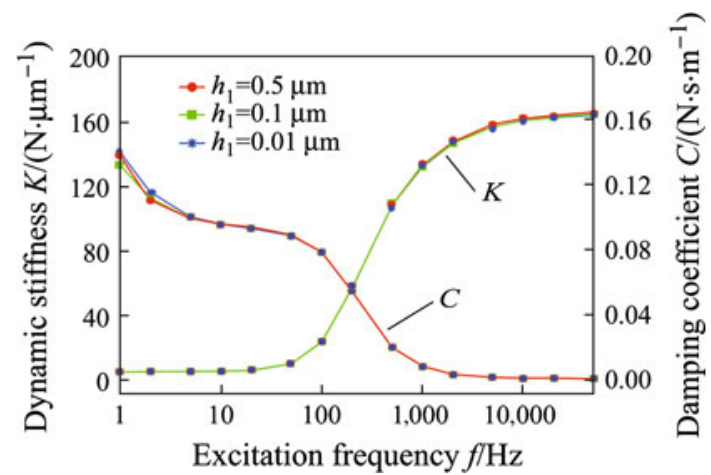

Fig. 6 Dynamic coefficients versus frequency with different excitation amplitudes $\left(h=10 \mu \mathrm{m}, P_{\mathrm{s}}=0.4 \mathrm{MPa}\right)$

Frequency dependence of the dynamic coefficients are clearly observed, i.e., with the increasing of excitation frequency $f$, the dynamic stiffness $K$ increases while the damping coefficient $C$ decreases, and this dependence is especially stronger from 100 to $1,000 \mathrm{~Hz}$. In addition, the excitation amplitude has little influence on the dynamic coefficients. Due to compressibility effects as mentioned in Sect. 2, in particular, the relationship between dynamic coefficients and excitation frequency is nonlinear. In the lower frequency range (below $10 \mathrm{~Hz}$ ), the dynamic stiffness is asymptotically equal to the static stiffness. Otherwise, effects of dynamic squeeze film become dominant and the dynamic stiffness of the bearing increases with increasing excitation frequency. This increase slows down until an asymptotic value is reached above about $10,000 \mathrm{~Hz}$, which is also due to air compressibility. Similarly, the damping is relatively larger at lower frequencies and is negligible beyond $1,000 \mathrm{~Hz}$.

The influence of the air gap height on dynamic characteristics of the aerostatic bearing is also quantitatively investigated by numerical calculations. Three air gap heights 10,20 and $30 \mu \mathrm{m}$ are considered, and the ratio 


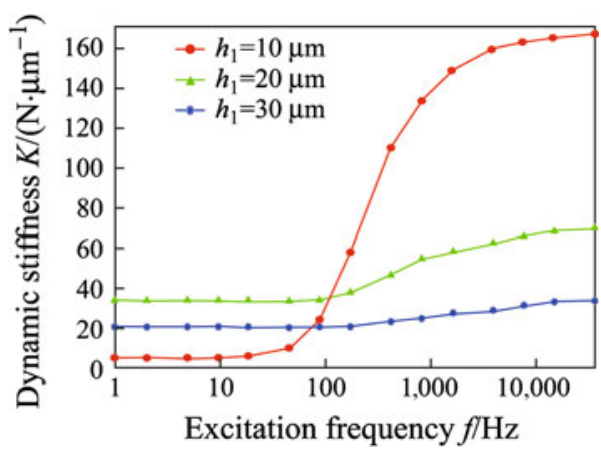

Fig. 7 Dynamic stiffness with various air gap heights $\left(P_{\mathrm{s}}=0.4 \mathrm{MPa}\right)$

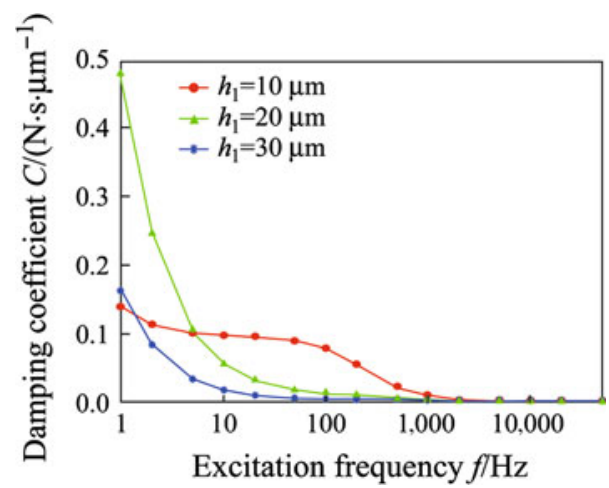

Fig. 8 Damping with various air gap heights $\left(P_{\mathrm{s}}=0.4 \mathrm{MPa}\right)$

$h_{1} / h$ is kept as a constant in each case. With different air gap heights, dynamic stiffness and damping coefficients of the bearing are plotted in Figs. 7 and 8, respectively. From Fig. 7, the dynamic stiffness reaches a maximum value at a certain air gap height in the low frequency range, which corresponds to the static stiffness of the bearing, while it becomes larger at smaller air gap heights in the high frequency range. The dynamic stiffness also tends to be independent of the excitation frequency at very small gap heights. From Fig. 8, the damping coefficient at a small excitation frequency (below $5 \mathrm{~Hz}$ ) is larger at a $20 \mu \mathrm{m}$ gap height than that at the other heights, and it declines faster to almost zero at larger air gap heights.

The influence of the air supply pressure is illustrated in Figs. 9 and 10. With the increase of supply pressure, dynamic stiffness and damping coefficients are increasing in the whole excitation frequency range. Moreover, variation of these dynamic coefficients with the excitation frequency is larger at higher supply pressure values.

\section{Conclusions}

This paper numerically investigates dynamic stiffness and damping characteristics of an ultra-precision aerostatic

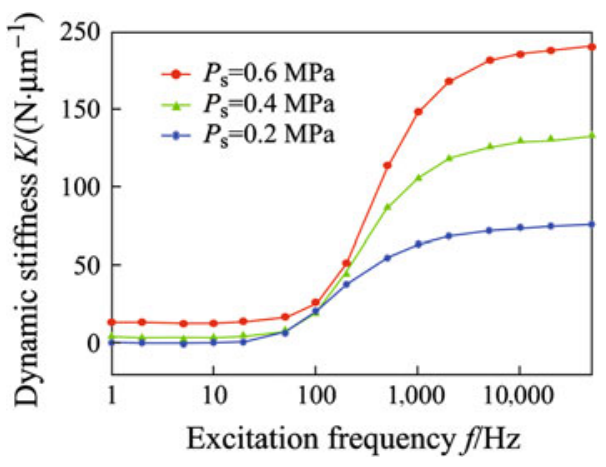

Fig. 9 Dynamic stiffness with various supply pressures $(h=10 \mu \mathrm{m})$

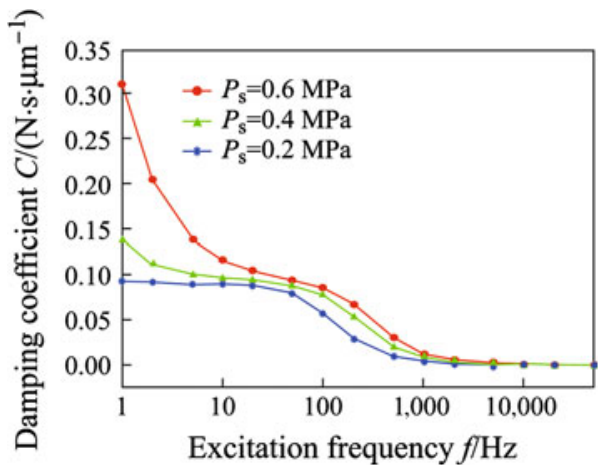

Fig. 10 Damping with various supply pressures $(h=10 \mu \mathrm{m})$

bearing under small air gap height perturbations. Nonlinear frequency dependence of these dynamic characteristics is revealed by a theoretical analysis, which is due to compressibility of the air. Furthermore, the numerical results demonstrate their behaviors, i.e., the dynamic stiffness increases while the damping decreases with increasing excitation frequency. At small bearing gap heights and high supply pressure, stronger frequency-dependent behaviors of dynamic characteristics of the bearing are observed. From these results, it is further confirmed that the frequency-dependent dynamic characteristics should be taken into account in the design of ultra-precision aerostatic bearings.

Acknowledgments This study was supported by the National Basic Research and Development Program of China (Grant No. 2009CB724205) and the National Natural Science Foundation of China (Grant Nos. 51121002, 51175196).

\section{References}

1. Fourka M, Tian Y, Bonis M (1996) Prediction of the stability of air thrust bearings by numerical, analytical and experimental methods. Wear 198(1/2):1-6

2. Mukai T (2006) Analysis of dynamic characteristics of air bearing. Nippon Steel Tech Rep 93:15-17 
3. Otsu Y, Miyatake M, Yoshimoto S (2011) Dynamic characteristics of aerostatic porous journal bearings with a surface restricted layer. J Tribol 133:1-10

4. Miyatake M, Yoshimoto S (2010) Numerical investigation of static and dynamic characteristics of aerostatic thrust bearings with small feed holes. Tribol Int 43:1353-1359

5. Arghir M, Matta P (2009) Compressibility effects on the dynamic characteristics of gas lubricated mechanical components. Comptes Rendus Mécanique 337(11/12):739-747
6. Bhat N, Kumar S, Tan W et al (2012) Performance of inherently compensated flat pad aerostatic bearings subject to dynamic perturbation forces. Precis Eng 36(3):399-407

7. Karniadakis G, Beskok A, Aluru N (2005) Microflows and nanoflows: fundamentals and simulation. Springer, New York 